\title{
The role and mechanism of WEE1 on the cisplatin resistance reversal of the HepG2/DDP human hepatic cancer cell line
}

\author{
WEIFENG ZHAO $^{1}$, SHUYUAN LIU ${ }^{1}$, QIAN DOU ${ }^{1}$, CHANGAN LI ${ }^{1}$, JINGPEI DU ${ }^{1}$ and WEIHUA REN ${ }^{2}$ \\ ${ }^{1}$ Department of Infections, The Third Affiliated Hospital of Xinxiang Medical University, Xinxiang, Henan 453003; \\ ${ }^{2}$ Central Laboratory, Luoyang Central Hospital Affiliated to Zhengzhou University, Luoyang, Henan 471009, P.R. China
}

Received September 20, 2014; Accepted May 20, 2015

DOI: 10.3892/ol.2015.3647

\begin{abstract}
Drug resistance to cisplatin with continuous drug treatment is one of the most common causes of chemotherapy failure in hepatic carcinoma. Accumulating evidence suggests that WEE1 G2 checkpoint kinase (WEE1) is involved in cisplatin resistance, which has been demonstrated to correlate with cancer initiation and progression. However, the role and molecular mechanism of WEE1 in the drug resistance of hepatic cancer remains unclear. In the present study, using the WEE-knockdown hepatic cancer cell line HepG2/DDP, the role of WEE1 and its molecular mechanism were investigated. It was demonstrated that silencing WEE1 expression resulted in an increased cisplatin sensitivity of HepG2/DDP, in addition to an increased rate of apoptosis and intracellular concentration of rhodamine 123. The expression levels of P-gp, MDR1, MRP1, LRP, BCL-2, survivin and GST in WEE1-silenced HepG2/DDP cells were significantly reduced, and phosphorylation levels of MEK and ERK were significantly downregulated. The results demonstrated that WEE1 negatively regulated the multidrug resistance potential of human hepatic cancer cells by modulating the expression of relevant drug resistance genes and the activity of the MEK/ERK pathway. Therefore, WEE1 may be a monitoring bio-marker for drug resistance, and a therapeutic target in hepatic cancer.
\end{abstract}

\section{Introduction}

Hepatic cancer is the most common malignancy and is responsible for the highest number of cancer-associated mortalities worldwide (1). Platinum-based agents are an important class of chemotherapy drugs for hepatic cancer, among which cisplatin is typical. This class of drugs are DNA alkylating agents that cross-link the DNA molecules to affect their replication, transcription and other normal functions, leading to cell growth arrest, apoptosis and death (2). Cisplatin is indicated for

Correspondence to: Professor Weihua Ren, Central Laboratory, Luoyang Central Hospital Affiliated to Zhengzhou University, 288 Zhongzhou Middle Road, Luoyang, Henan 471009, P.R. China E-mail: ren-wei-hua@163.com

Key words: WEE1, cisplatin resistance, hepatic cancer patients with advanced inoperable diseases, and also as part of comprehensive therapeutic regimens, including surgical operation (3). However, tumor cells tend to develop drug resistance to cisplatin with continuous drug treatment, resulting in treatment failure (4). At this point, the only option for these patients is to select other chemotherapeutic drugs, despite their limited clinical benefits. One of the reasons for the limited benefits of other drugs is that tumor cells are likely to develop resistance to the other chemotherapeutic drugs as they develop resistance to cisplatin; this is called multidrug resistance (5). Several key regulatory proteins have been identified in the past decade as a result of the considerable progress in the biological studies of tumorigenesis and tumor progression; and a number of successes have been achieved regarding the therapies targeting these proteins in clinical practice (6). These key proteins, including WEE1 G2 checkpoint kinase (WEE1), regulate tumor cell growth and metastasis, and serve a role in the development of drug resistance in tumor cells. WEE1 is a cell cycle-associated kinase (7) and has been demonstrated to be expressed in certain cisplatin-resistant cells from human epidermal carcinoma (8). To investigate the role of WEE1 in the cisplatin-resistant hepatic cancer cell line HepG2/DDP, the current study examined the reversal of cisplatin resistance in HepG2/DDP by silencing WEE1, and explored the mechanisms of triptolide-mediated reversal of drug resistance by probing the aspects associated with the mechanisms of tumor drug resistance.

\section{Materials and methods}

Cells and cell culture. The multidrug resistant human hepatic cancer cell line HepG2/DPP was obtained from The Third Affiliated Hospital of Xinxiang Medical University (Xinxiang, China) and cultured with RPMI 1640 (containing 10\% calf serum; Corning Life Sciences, Manassas, VA, USA) in an incubator under $37^{\circ} \mathrm{C}, 5 \% \mathrm{CO}_{2}$ and saturated humidity conditions. Trypsin-EDTA $[0.25 \%$ in phosphate-buffered saline (PBS); Corning Life Sciences] was used for digestion and passaging. Cells in the logarithmic growth phase were used in all experiments. The present study was approved by the ethics committee of Luoyang Central Hospital Affiliated to Zhengzhou University (Luoyang, China).

Silencing of WEEl expression with lentivector-weel short hairpin (sh)RNA. The cells were cultured in 24-well 
plates, at a density of $3 \times 10^{4}$ cells/well. The culture was continued until $\sim 70 \%$ cells reached confluence. The weel shRNA (h) viral vectors (Lentiviral Particles; Santa Cruz Biotechnology, Inc., Santa Cruz, CA, USA) were prepared in accordance with the procedures described in the manufacturer's instructions. The cells were transfected with different multiplicity of infection (MOI) values (10 and 20). The culture medium was switched to virus-free medium (PT3414-1; Corning Life Sciences) $24 \mathrm{~h}$ following transfection. The culture was continued for $48 \mathrm{~h}$ for passaging. The silencing status of WEE1 was evaluated by western blotting and quantitative polymerase chain reaction (qPCR). The HepG2/DDP cells were the parental control group, the HepG2/DDP cells transfected with blank vector were the negative control group and the HepG2/DDP cells transfected with WEE1 short hairpin (sh)RNA were the shRNA\#1 (MOI, 10) and shRNA\#2 (MOI, 20) silencing groups.

Determination of cell sensitivity to cisplatin with MTS assay. Cells $\left(5 \times 10^{4}\right.$ cells $\left./ \mathrm{ml}\right)$ were seeded in 96-well microplates, at $100 \mu \mathrm{l} /$ well and cultured overnight to allow cell adherence. Next, different concentrations of cisplatin $(0,30,60,90,120$, 150, 180, 210, 240, 270 and $300 \mu \mathrm{M}$; Sigma-Aldrich, St. Louis, MO, USA) were added and the culture was continued for $72 \mathrm{~h}$. Following aspiration of the culture medium, $50 \mu \mathrm{l}$ MTS (Promega Corporation, Madison, WI, USA) was added in accordance with the reagent instructions and cultured for $4 \mathrm{~h}$. The optical density (OD) was measured at $490 \mathrm{~nm}$ wavelength with a Bio-Rad 3550 microplate reader (Bio-Rad Laboratories, Inc., Hercules, CA, USA) and the inhibition rate of the drug on the cells was calculated as follows: Inhibition rate $=(1-\mathrm{OD}$ of experimental group/OD of control group) $x 100$. Using the cisplatin concentration as abscissa and the inhibition rate as ordinate, the inhibition curve was plotted and fitted to obtain the half maximal inhibitory concentration $\left(\mathrm{IC}_{50}\right)$. Reversal fold (RF) was defined as $\mathrm{RF}=\mathrm{IC}_{50}$ (silence group) $/ \mathrm{IC}_{50}$ (parental group).

Determination of intracellular rhodamine-123 (Rh-123) content, cell surface P-glycoprotein $(P-g p)$ expression, apoptosis and caspase $-3 / 8$ activity with flow cytometry. Following addition of $100 \mu \mathrm{l}$ of $10 \mu \mathrm{M}$ Rh-123 staining solution (Beyotime Institute of Biotechnology, Shanghai, China), the cells in logarithmic growth phase were cultured for $1 \mathrm{~h}$ and harvested. The fluorescence intensity of intracellular Rh-123 was detected with a flow cytometer (FACSAria ${ }^{\mathrm{TM}}$ I; BD Biosciences, Franklin Lakes, NJ, USA) to indicate intracellular Rh-123 content. P-gp expression was determined with flow cytometry using specific procedures as follows: $10^{6}$ cells were added to $0.1 \mathrm{ml}$ culture medium for each sample and stained in accordance with the kit instructions [Anti-P-gp/fluorescein isothiocyanate (FITC) fluorescent antibody kit; Abcam, Cambridge, MA, USA]; cell fluorescence intensity was detected with a flow cytometer to indicate the P-gp expression level. Apoptosis was detected using the annexin V-FITC/propidium iodide (PI; BD Biosciences, Franklin Lakes, NJ, USA) double-staining technique; the cells were treated with $20 \mu \mathrm{M}$ cisplatin for $24 \mathrm{~h}$ and harvested to determine apoptosis in accordance with the manufacturer's instructions. Following similar cisplatin treatment, intracellular caspase- 3 and -8 activities were determined with phycoerythrin-labeled anti-active rabbit IgG caspase-3 (cat no. sc-7147) and -8 antibodies (cat no. sc-7890; 1:1,000 dilution; Santa Cruz Biotechnology, Inc.) using the flow cytometer in accordance with the manufacturer's instructions.

Determination of protein levels of WEE1 G2 checkpoint kinase (WEE1), multidrug resistance protein 1 (MDR1), multidrug resistance associated protein 1 (MRP1), lipoprotein receptor-related protein ( $L R P), B$-cell lymphoma 2 (BCL-2), survivin and glutathione $S$-transferase (GST); and phosphorylation levels of mitogen-activated protein kinase kinase $(M E K)$ and extracellular-signal-regulated kinase (ERK) in HepG2/DPP cells using western blotting. Cells in the logarithmic growth phase were lysed using a cell lysis kit (Beyotime Institute of Biotechnology) to extract the total protein content. The proteins were separated in 12\% SDS-PAGE (Beyotime Institute of Biotechnology) at $220 \mathrm{~V}$ and transferred onto a polyvinylidene difluoride (PVDF) membrane (Beyotime Institute of Biotechnology). Antibodies for WEE1 (cat no. sc-9037; rabbit anti-human IgG; 1:2,000 dilution), MDR1 (cat no. sc-55510; mouse anti-human IgG; 1:2,000 dilution), MRP1 (cat no. sc-13960; rabbit anti-human IgG; 1:2,000 dilution), LRP (cat no. sc-390134; mouse anti-human IgG; 1:2,000 dilution), BCL-2 (cat no. sc-492; rabbit anti-human IgG; 1:2,000 dilution), survivin (cat no. sc-10811; rabbit anti-human IgG; 1:2,000 dilution), GST (cat no. sc-459; rabbit anti-human IgG; 1:2,000 dilution), p-MEK (cat no. sc-130203; rabbit anti-human $\mathrm{IgG}$; 1:1,500 dilution) and p-ERK (cat no. sc-13073; rabbit anti-human $\mathrm{IgG}$; 1:1,500 dilution) were used to detect the target proteins, and $\beta$-actin (cat no. sc-1616; goat anti-human IgG; 1:5,000 dilution) and ERK (cat no. sc-94; rabbit anti-human $\mathrm{IgG} ; 1: 2,000$ dilution) were used as the internal reference genes. All antibodies were purchased from Santa Cruz Biotechnology, Inc. Cells were incubated with the antibodies at $4^{\circ} \mathrm{C}$ overnight. Subsequent to washing off the primary antibody with $10 \% \mathrm{PBS}$, horseradish peroxidase (HRP)-conjugated secondary antibody (cat no. sc-2005; goat anti-mouse IgG-HRP; 1:2,000 dilution; and cat no. sc-2004; goat anti-rabbit IgG-HRP; 1:2000 dilution) was added to the cells and incubated for $1 \mathrm{~h}$. Cells were washed with $10 \%$ PBS, then an enhanced chemiluminescence (ECL) kit (Millipore, Boston, MA, USA) was used to identify the immunoreactive bands using methods according to the manufacturer's instructions.

Determination of the MRNA levels of WEE1, MDR1, MRP1, $L R P, B C L-2$, survivin and GST in HepG2/DPP tumor cells using reverse transcription $(R T)-q P C R$. Following the extraction of total RNA from each group of cells in the logarithmic growth phase using TRIzol (Invitrogen Life Technologies, Carslbad, CA, USA), RT was conducted with Real-Time PCR kit (Ambion, Austin, TX, USA) to obtain the cDNA. The primer sequences were as follows: WEE1, F 5'-GATGAGCAGAACGCTTTGAGAG-3' and R 5'-CAG AGGCAGCATTTGGGATT-3'; MDR1, F 5'-AAAAAG ATCAACTCGTACCACTC-3' and R 5'-GCACAAAAT ACACCAACAA-3'; MRP1, F 5'-ACTTCCACATCTGCT TCGTCAGTG-3' and R 5'-ATTCAGCCACAGGAGGTA GAGAGC-3'; LRP, F 5'-AGTCAGAAGCCGAGAAAG-3' and R 5'-CCCAGCCACAGCAAGGT-3'; BCL-2, F 5'-ACG GGGTGAACTGGGGGAGGA-3' and R 5'-TGTTTGGGG 
A

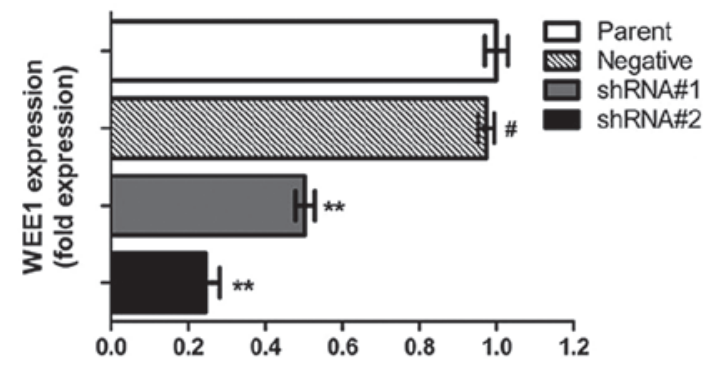

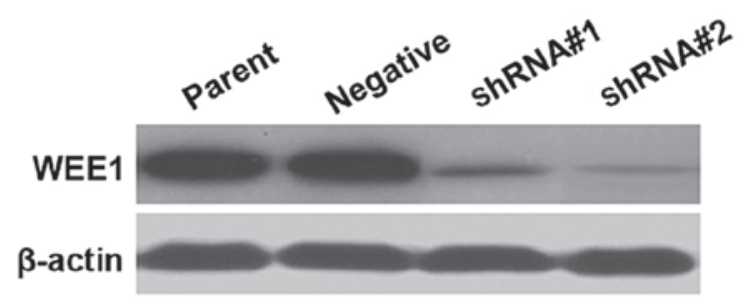

Figure 1. Lentivector-wee1 shRNA significantly downregulated WEE1 expression in HepG2/DDP cells. (A) RT-qPCR assay indicated reduced mRNA expression levels of WEE1 in the shRNA\#1 and shRNA\#2 groups compared with the parental and negative groups. Data are presented as the mean \pm standard deviation. ${ }^{~} \mathrm{P}>0.05$ and ${ }^{* *} \mathrm{P}<0.05$ vs. the parental group, $\mathrm{n}=5$. (B) Western blot assay demonstrated that there were reduced levels of WEE1 protein in WEE1-silenced shRNA\#1 and shRNA\#2 groups compared with the parental and negative groups. $\beta$-actin was used as an internal control. WEE1, WEE1 G2 checkpoint kinase; shRNA, short hairpin RNA; shRNA\#1, WEE1-silenced group 1; shRNA\#2, WEE1-silenced group 2.

A

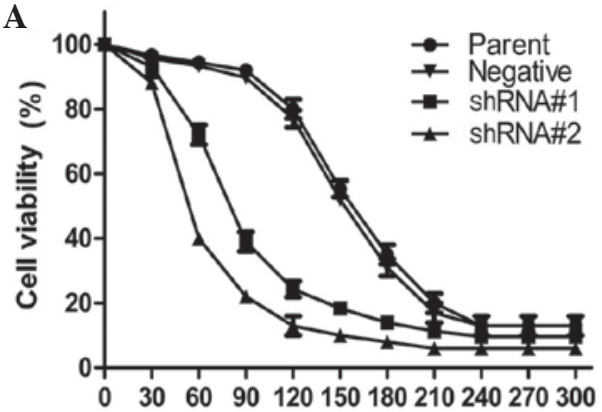

C

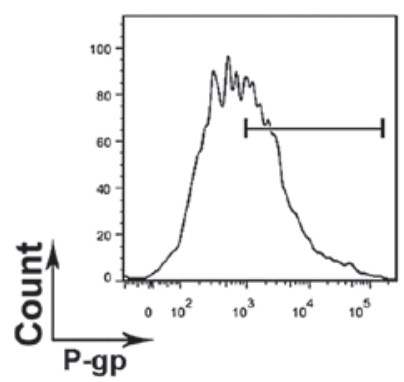

Negative

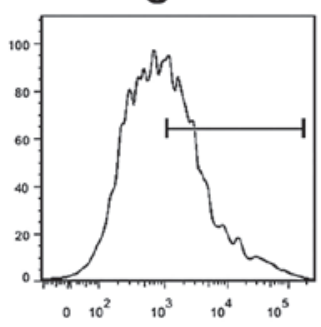

B

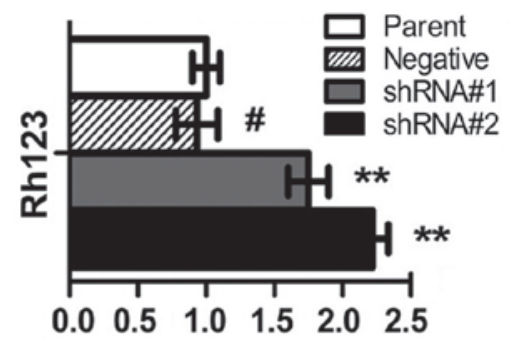

D Parent

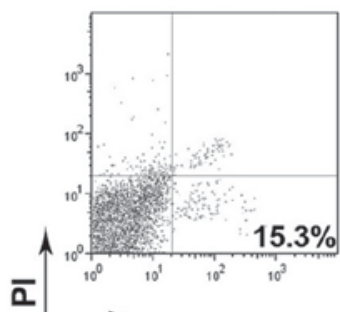

Negative

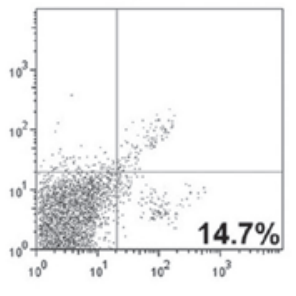

ShRNA\#1

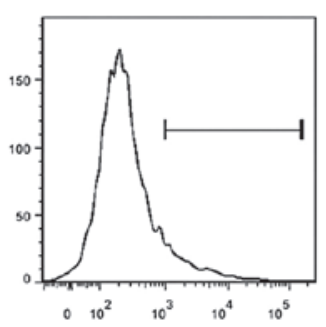

shRNA\#2

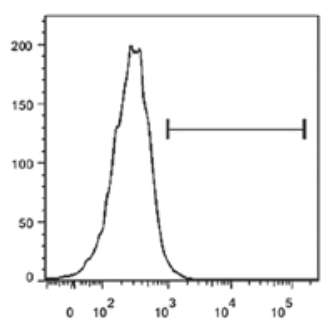

Annexin V-FITC

Figure 2. WEE1 silencing increased the cisplatin sensitivity of HepG2/DPP cells. (A) MTS assay demonstrated that there was an increased cisplatin sensitivity in the shRNA\#1 and shRNA\#2 groups compared with the parental and negative groups. (B) Fluorescence assay demonstrating increased levels of Rh123 in the shRNA\#1 and shRNA\#2 groups compared with the parental and negative groups. (C) Flow cytometry assay demonstrated a decreased P-gp content in the shRNA\#1 and shRNA\#2 groups compared with the parental and negative groups. (D) Flow cytometry assay demonstrated that there were increased levels of cell apoptosis in the shRNA\#1 and shRNA\#2 groups compared with the parental and negative groups. Data are presented as the mean \pm standard deviation. ${ }^{\#} \mathrm{P}>0.05$ and ${ }^{* *} \mathrm{P}<0.05$ vs. the parental group, $\mathrm{n}=3$. shRNA, short hairpin RNA; shRNA\#1, WEE1-silenced group 1; shRNA\#2, WEE1-silenced group 2; Rh123, rhodamine 123; PI, propidium iodide; FITC, fluorescein isothiocyanate; P-gp,P-glycoprotein. 
A

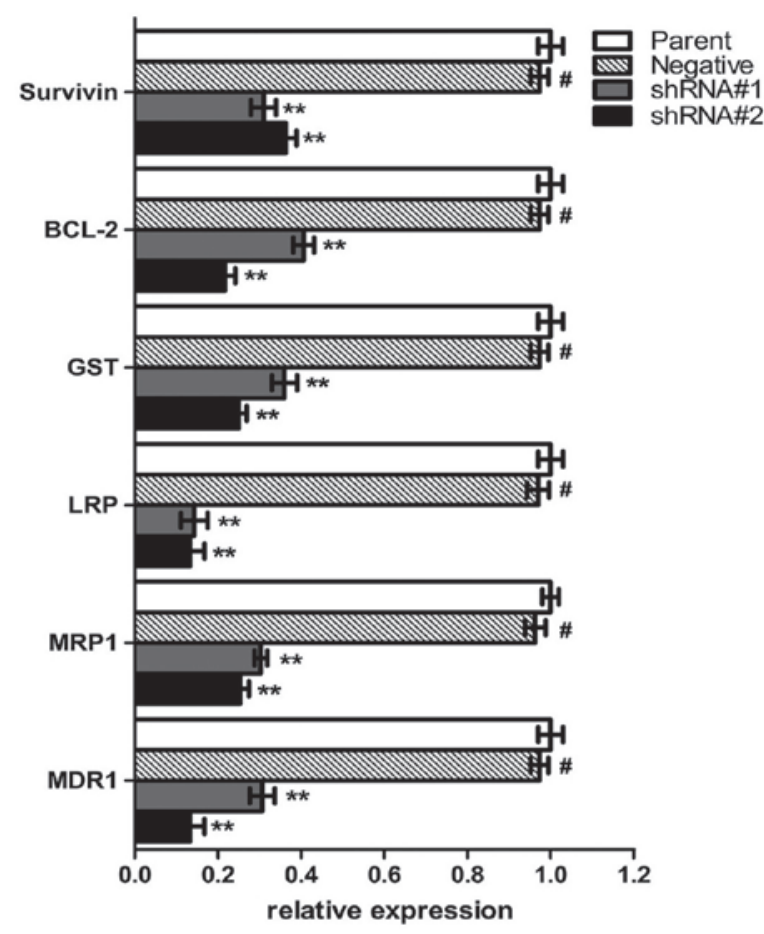

B
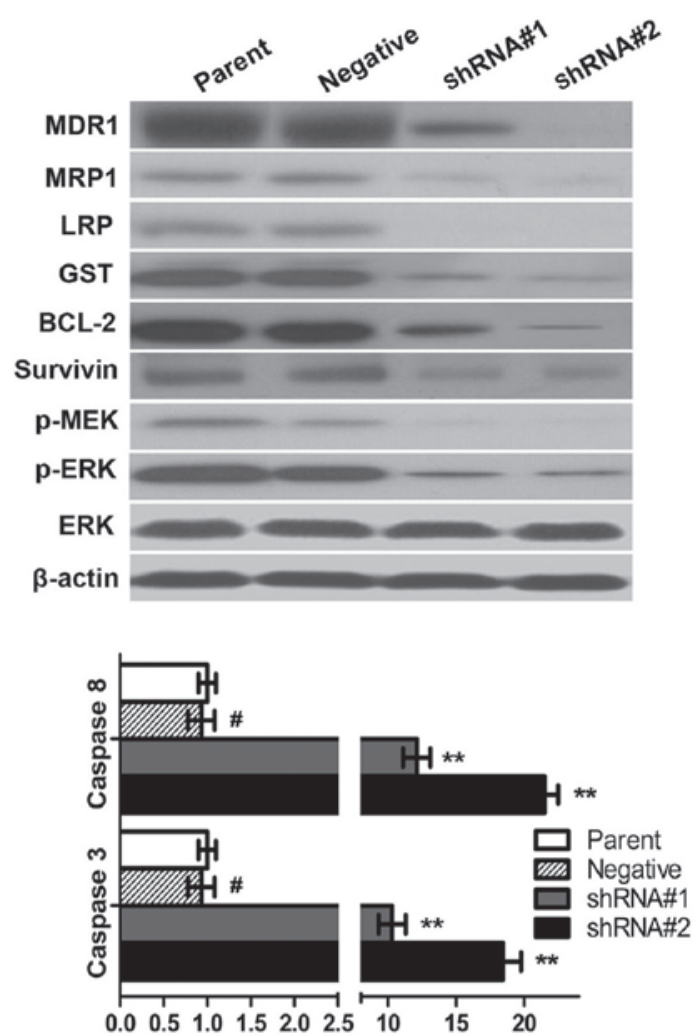

C
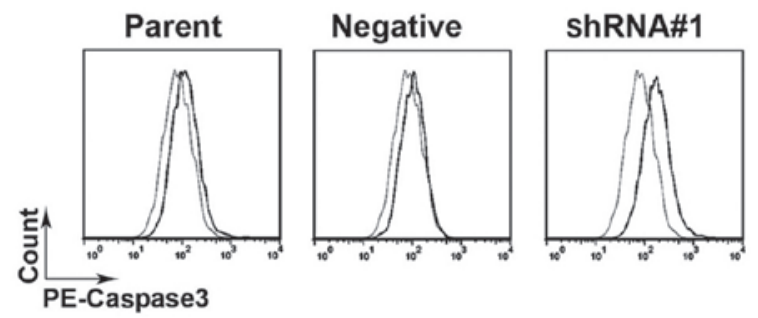

ShRNA\#2

D

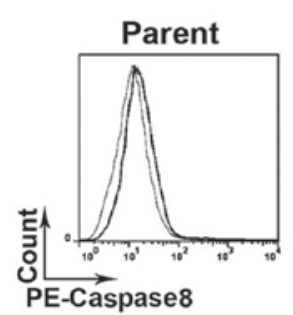

Negative

ShRNA\#1
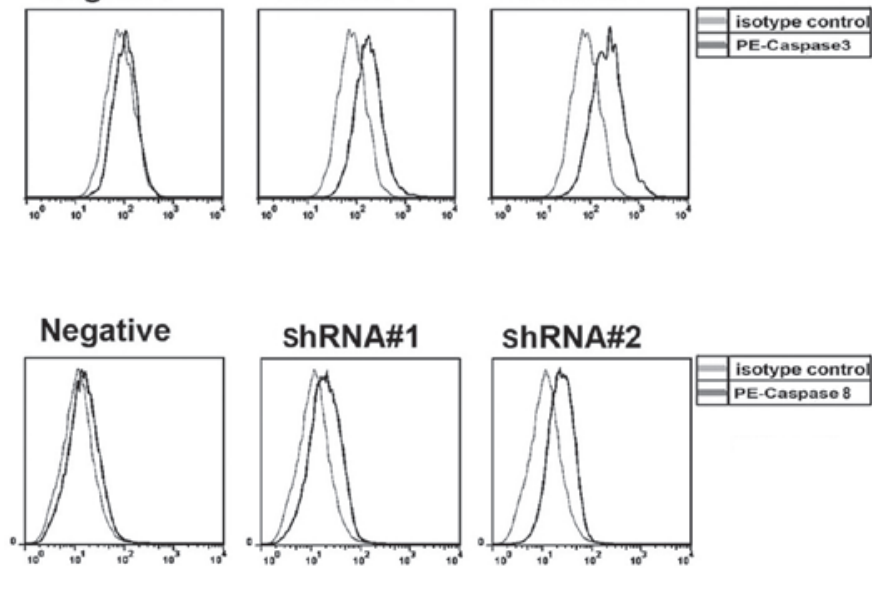

ShRNA\#2

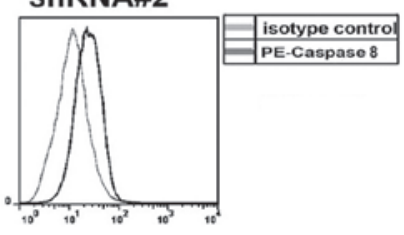

Figure 3. WEE1 silencing downregulated the expression of drug resistance-associated genes in HepG2/DPP cells. (A) Reverse transcription-quantitative polymerase chain reaction demonstrated decreased mRNA expression levels of survivin, BCL-2, GST, LRP, MRP1 and MDR1 in the shRNA\#1 and shRNA\#2 groups compared with parental and negative groups. Data are presented as the mean \pm standard deviation. ${ }^{\prime \prime} \mathrm{P}>0.05$ and ${ }^{* * *} \mathrm{P}<0.05$ vs. the parental group, $\mathrm{n}=5$. (B) Western blot assay demonstrated reduced expression levels of survivin, BCL-2, GST, LRP, MRP1, MDR1 p-MEK, p-ERK in the shRNA\#1 and shRNA\#2 groups compared with the parental and negative groups. $\beta$-actin and ERK were used as internal controls. (C and D) Flow cytometry demonstrated an increased activity of caspase-3/8 in the shRNA\#1 and shRNA\#2 groups compared with the parental and negative groups. Data are presented as the mean \pm standard deviation. ${ }^{*} \mathrm{P}>0.05$ and ${ }^{* * *} \mathrm{P}<0.05$ vs. the parental group, $\mathrm{n}=3$. shRNA, short hairpin RNA; shRNA\#1, WEE1-silenced group 1; shRNA\#2, WEE1-silenced group 2; BCL-2, B-cell lymphoma 2; GST, glutathione S-transferase; LRP, lipoprotein receptor-related protein; MRP1, multidrug resistance associated protein 1; MDR1, multidrug resistance protein 1; p-, phosphorylated; MEK, mitogen-activated protein kinase kinase; ERK, extracellular-signal-regulated kinase; PE, phycoerythrin.

3 min, 40 cycles of amplification were conducted under the following conditions: $95^{\circ} \mathrm{C}$ for $5 \mathrm{sec}, 65^{\circ} \mathrm{C}$ for $35 \mathrm{sec}, 72^{\circ} \mathrm{C}$ for $60 \mathrm{sec}$, and extension at $72^{\circ} \mathrm{C}$ for $5 \mathrm{~min}$.

Statistical analysis. The experimental data are presented as the mean \pm standard deviation and analyzed with SPSS software, version 13.0 (SPSS, Inc., Chicago, IL, USA). One-way analysis of variance was used for comparison; $\mathrm{P}<0.05$ was considered to indicate a statistically significant difference.

\section{Results}

Lentivector-weel shRNA significantly downregulated WEE1 expression in Hep G2/DDP cells. Transfection of HepG2/DDP 
cells with different MOI values resulted in varying degrees of WEE1 silencing. Subsequent to screening, cells transfected with the MOI values of 10 and 20 were selected for subsequent studies. As presented in Fig. 1, WEE1 expression levels in the shRNA\#1 (MOI, 10) and shRNA\#2 (MOI, 20) groups were 53 and $22 \%$ of the parental group cells, respectively $(\mathrm{P}<0.05)$. Blank vector shRNA cells (negative group) were observed to have no effect on WEE1 expression levels $(\mathrm{P}>0.05)$.

WEE1 silencing increased the sensitivity of HepG2/DPP cells to cisplatin. MTS assay results (Fig. 2A) demonstrated that the $\mathrm{IC}_{50}$ of cisplatin-mediated inhibition on HepG2/DPP cell proliferation was $156 \mu \mathrm{M}$, while the $\mathrm{IC}_{50} \mathrm{~s}$ for the shRNA\#1 and shRNA\#2 groups were 70.2 and $43.5 \mu \mathrm{M}$, respectively, suggesting that silencing WEE1 enabled an increase in the sensitivity of HepG2/DPP cells to cisplatin. The RFs calculated based on the $\mathrm{IC}_{50} \mathrm{~s}$ for shRNA\#1 and shRNA\#2 groups were 2.2 and 3.6 fold, respectively.

WEE1 silencing increased Rh-123 content, downregulated P-gp expression, and increased the rate of apoptosis and caspase-3/8 activity in HepG2/DPP cells. The fluorescence intensity of intracellular Rh-123 in the shRNA\#1 and shRNA\#2 groups increased by 1.75 - and 2.23-fold compared with the parental control group (Fig. 2B). By contrast, P-gp expression was downregulated to 73.2 and $34.5 \%$ of the control group, respectively (Fig. 2C), suggesting that elevation of intracellular Rh-123 was associated with the downregulation of P-gp, which may increase intracellular cisplatin. In addition, following treatment with $20 \mu \mathrm{M}$ cisplatin for $24 \mathrm{~h}$, the apoptosis rate of the HepG2/DDP parental group was $15.3 \%$, while those of the shRNA\#1 and shRNA\#2 groups were 28.4 and $39.8 \%$ (Fig. 2D; $\mathrm{P}<0.05$ ).

WEE1 silencing downregulated the expression of drug resistance-associated genes and the phosphorylation of MEK and ERK, and upregulated the activity of caspase-3/8 in HepG2/DPP cells. RT-qPCR analysis demonstrated that the gene transcription levels of MDR1, MRP1, LRP and GST decreased significantly following WEE1 silencing (Fig. 3A). Western blot results demonstrated that, compared with the control group, the protein levels were also reduced (Fig. 3B). These results indicate that the downregulation of protein expression was regulated at the transcriptional level.

Since WEE1 silencing enhanced apoptosis, the expression of 2 anti-apoptotic proteins, BCL-2 and survivin, were assessed. Western blotting indicated that WEE1 silencing was accompanied by downregulation of BCL-2 and survivin expression. RT-qPCR analysis demonstrated that the downregulation of expression was achieved at transcriptional level $(\mathrm{P}<0.05)$.

In order to explain these results, the MEK/ERK pathway was considered as the possible intracellular signaling pathway regulated by WEE1. Western blotting demonstrated that levels of phosphorylated MEK and ERK decreased following WEE1 silencing, indicating that the activity of this pathway was significantly suppressed.

Furthermore, the shRNA\#1 and shRNA\#2 groups were treated with $20 \mu \mathrm{M}$ cisplatin for $24 \mathrm{~h}$, and the activated caspase- 3 content was increased by 10.3 and 18.7-fold, respectively, compared with the control group; the activated caspase- 8 content increased 12.1 and 21.5-fold, compared with the control group (Fig. 3).

\section{Discussion}

In the present study, WEE1-silenced HepG2/DDP cell lines were constructed using the lentiviral vector transfection technique and then 2 representative cell lines with different degrees of WEE1 silencing were selected for subsequent studies. Previous studies have demonstrated the correlation between WEE1 and the resistance to certain tumor drugs $(9,10)$. The MTS assay results of the present study confirmed that WEE1 silencing increased the sensitivity of HepG2/DDP to cisplatin. Further experiments demonstrated that WEE1 silencing resulted in the elevation of intracellular $\mathrm{Rh}-123$ concentration and apoptosis. The 2 main mechanisms for tumor cells in the development of resistance to drugs include the overexpression of a variety of multidrug resistance genes to enhance drug efflux and lower intracellular drug concentration, and the resistance to drug-induced apoptosis (11). Thus, the current study hypothesized that WEE1 also reversed the drug resistance of HepG2/DDP through the regulation of these 2 drug resistance mechanisms.

To assess this hypothesis, the effects of WEE1 silencing on the expression of various multidrug resistant proteins were detected. The expression of cell surface P-gp was examined with flow cytometry; the results demonstrated that its expression was significantly downregulated. In addition, MDR1 and MRP1 expression levels were detected with western blotting; the results demonstrated that these proteins were also significantly downregulated. These 2 proteins belong to the ABC super-family and are able to pump chemotherapeutic drugs out of the cells (12). Furthermore, the present study also demonstrated that LRP protein expression was downregulated. Although LRP is not a member of the ABC family, it is also able to pump drugs out of the cells (13). We hypothesized that downregulation of the expression of these proteins may increase the concentration of intracellular chemotherapeutic drugs. This is consistent with the elevation of intracellular drug concentration of Rh-123 observed in the present study. RT-qPCR analysis demonstrated that the regulation of these expression levels was achieved at the transcriptional level.

The effects of WEE1 suppression on apoptosis-associated proteins was also analyzed. BCL-2 and survivin are two common anti-apoptotic proteins (14). It has previously been demonstrated that the BCL-2 protein expression level is significantly higher in drug-resistant HepG2/DDP cells compared with non-drug-resistant mother cells (15); this was confirmed in the current study. Western blotting results indicated that BCL-2 and survivin levels declined significantly following WEE1 silencing; RT-qPCR analysis demonstrated that the regulation of these expression levels was achieved at the transcriptional level. Flow cytometry analysis demonstrated that WEE1 silencing enhanced apoptosis and also significantly increased caspase-3/8 activity, clarifying the pathway of apoptosis.

Tumor cells can also develop resistance to chemotherapeutic drugs through another mechanism by which chemotherapeutic drugs are inactivated in metabolism. For example, GST can acylate and inactivate cisplatin $(16,17)$. The current study 
demonstrated that GST levels significantly increased in HepG2/DDP cells and significantly decreased subsequent to WEE1 silencing, suggesting that GST was also involved in WEE1-mediated drug resistance to cisplatin.

Since WEE1 is able to interact with multiple cell signaling pathways (7), the present study assessed the key ERK/MEK signaling pathway, which is closely associated with cell growth (18) and drug resistance to cisplatin (19). Western blotting demonstrated that ERK and MEK phosphorylation levels significantly decreased with the silencing of WEE1, suggesting that the activity of this signaling pathway declined and it was involved in WEE1-mediated cisplatin drug resistance.

In summary, the current study demonstrated that silencing WEE1 is able to reverse the multidrug resistance of HepG2/DDP cells. The mechanisms of action may be associated with the downregulation of multidrug resistance genes to inhibit drug efflux, the enhancement of apoptosis, and the inhibition of MEK/ERK pathway activity.

\section{References}

1. Zhu Y, Zhu L, Lu L, Zhang L, Zhang G, Wang Q and Yang P: Role and mechanism of the alkylglycerone phosphate synthase in suppressing the invasion potential of human glioma and hepatic carcinoma cells in vitro. Oncol Rep 1: 431-436, 2014.

2. Kelland L: The resurgence of platinum-based cancer chemotherapy. Nat Rev Cancer 7: 573-584, 2007.

3. Brock MV, Hooker CM, Syphard JE, Westra W, Xu L, Alberg AJ, Mason D, Baylin SB, Herman JG, Yung RC, et al: Surgical resection of limited disease small cell lung cancer in the new era of platinum chemotherapy: Its time has come. J Thorac Cardiovasc Surg 129: 64-72, 2005.

4. Zhao L, Li W, Zhou Y, Zhang Y, Huang S, Xu X, Li Z and Guo Q: The overexpression and nuclear translocation of Trx-1 during hypoxia confers on HepG2 cells resistance to DDP, and GL-V9 reverses the resistance by suppressing the Trx-1/Ref-1 axis. Free Radic Biol Med 82: 29-41, 2015.

5. Shen DW, Pouliot LM, Hall MD and Gottesman MM: Cisplatin resistance: A cellular self-defense mechanism resulting from multiple epigenetic and genetic changes. Pharmacol Rev 64: 706-721, 2012

6. Mitsudomi T: Advances in target therapy for lung cancer. Jpn J Clin Oncol 40: 101-106, 2010.

7. McCarthy N: Cell cycle: A WEE pointer. Nat Rev Cancer 12 : 378-379, 2012

8. Pouliot LM, Chen YC, Bai J, Guha R, Martin SE, Gottesman MM and Hall MD: Cisplatin sensitivity mediated by WEE1 and CHK1 is mediated by miR-155 and the miR-15 family. Cancer Res 72: 5945-5955, 2012.
9. Kogiso T, Nagahara H, Hashimoto E, Ariizumi S, Yamamoto M and Shiratori K: Efficient induction of apoptosis by weel kinase inhibition in hepatocellular carcinoma cells. PLoS One 6: e100495, 2014.

10. Tsai SC, Yang JS, Peng SF, Lu CC, Chiang JH, Chung JG, Lin MW, Lin JK, Amagaya S, Wai-Shan Chung C, et al: Bufalin increases sensitivity to AKT/mTOR-induced autophagic cell death in SK-HEP-1 human hepatocellular carcinoma cells. Int J Oncol 4: 1431-1442, 2012.

11. de Figueiredo-Pontes LL, Pintão MC, Oliveira LC, Dalmazzo LF, Jácomo RH, Garcia AB, Falcão RP and Rego EM: Determination of P-glycoprotein, MDR-related protein 1, breast cancer resistance protein, and lung-resistance protein expression in leukemic stem cells of acute myeloid leukemia. Cytometry B Clin Cytom 74: $163-168,2008$.

12. Fridman E, Skarda J, Pinthus JH, Ramon J and Mor Y: Expression of multidrug resistance-related protein (MRP-1), lung resistance-related protein (LRP) and topoisomerase-II (TOPO-II) in Wilms' tumor: Immunohistochemical study using TMA methodology. Biomed Pap Med Fac Univ Palacky Olomouc Czech Repub 152: 47-51, 2008.

13. Kerr EH, Frederick PJ, Egger ME, Stockard CR, Sellers J, DellaManna D, Oelschlager DK, Amm HM, Eltoum IE, Straughn JM, et al: Lung resistance-related protein (LRP) expression in malignant ascitic cells as a prognostic marker for advanced ovarian serous carcinoma. Ann Surg Oncol 20: 3059-3065, 2013.

14. Mita AC, Mita MM, Nawrocki ST and Giles FJ: Survivin: Key regulator of mitosis and apoptosis and novel target for cancer therapeutics. Clin Cancer Res 14: 5000-5005, 2008.

15. Li K, Chen B, Xu L, Feng J, Xia G, Cheng J, Wang J, Gao F and Wang X: Reversal of multidrug resistance by cisplatin-loaded magnetic Fe3O4 nanoparticles in A549/DDP lung cancer cells in vitro and in vivo. Int J Nanomedicine 8: 1867-1877, 2013.

16. Surowiak P, Materna V, Kaplenko I, Spaczyński M, Dietel M, Lage $\mathrm{H}$ and Zabel M: Augmented expression of metallothionein and glutathione S-transferase pi as unfavourable prognostic factors in cisplatin-treated ovarian cancer patients. Virchows Arch 447: 626-633, 2005.

17. Zhu Y, Liu XJ, Yang P, Zhao M, Lv LX, Zhang GD, Wang Q and Zhang L: Alkylglyceronephosphate synthase (AGPS) alters lipid signaling pathways and supports chemotherapy resistance of glioma and hepatic carcinoma cell lines. Asian Pac J Cancer Prev 7: 3219-3226, 2014

18. Montagut $\mathrm{C}$ and Settleman J: Targeting the RAF-MEK-ERK pathway in cancer therapy. Cancer Lett 283: 125-134, 2009.

19. Brozovic A and Osmak M: Activation of mitogen-activated protein kinases by cisplatin and their role in cisplatin-resistance. Cancer Lett 251: 1-16, 2007. 